\title{
ON
}

\section{RESECTION OF PORTIONS OF INTESTINE.}

\author{
$\mathbf{B Y}$ \\ FREDERICK TREVES, F.R.C.S., \\ ASSISTANT SURGEON TO, AND SENIOR DEMONSTRATOR OF ANATOMY AT, \\ THE LONDON HOSPITAL.
}

(Received October 5th-Read December 12th, 1882.)

THE operation of resection of certain portions of the bowel for various diseased conditions has now been performed so many times and with such a proportion of success that surgeons are in a position, I think, to consider not only the justifiability of the procedure, but also the circumstances that best conduce to its successful issue.

Indeed, from an examination of the cases already published, it would appear that the operation is not only justifiable, but that in selected and proper instances it is the best and perhaps the only means of affording substantial relief or reliable cure. Resection has now been practised upon all parts of the intestinal canal from the pylorus to the rectum, but I will confine my remarks to the principal difficulties and risks of the operation, and endeavour to point out how those difficulties may be met, concluding with an account of a case in which I recently resected a portion of the descending colon by a new method. A careful examination of the recorded cases of resection, and 
especially of the fatal cases, reveals one very striking fact, and that is that the failure of the operation is more often due to defects in technical details than to any other cause. The future of the operation, and the probability of its being established as a proper and sound surgical procedure, depend, I think, at the present moment, more upon improvement in the details of the operation itself than upon a more elaborate knowledge of the circumstances under which it should be performed.

In discussing the items of the operation I will consider first resection of the small gut, and subsequently resection of the colon, omitting altogether the operations for pyloric cancer and for artificial anus.

In resecting portions of the small intestine the abdomen is opened in the middle line below the umbilicus. When the peritoneum has been divided, and the diseased part is being searched for, some protusion of the distended gut is very usual. It is most desirable that this protrusion should be prevented. With care the intestines can be retained by the application of large carbolised sponges, and by puncturing the distended bowel in several places with a capillary trochar. This pricking I have practised without ill result in three cases of laparotomy for intestinal obstruction. When the diseased part has been dragged out of the wound, it becomes necessary to control the gut above and below to prevent extravasation of fæces when the resection is performed. This control of the bowel has been effected in many ways. By some it is advised that a partial union of the bowel should be effected before the preparations for an intestinal fistula are made. Of the otber methods available there are Jobert's invagination process, which consists of invaginating the upper into the lower end of the completely divided intestine and then stitching together the serous surfaces, and Maisonneuve's plan of "intestinal anastomosis," where the divided small gut above the obstruction is joined to some part of the colon. Neither of these processes, however, would appear to have been applied to the human subject. There remains 
the method of directly uniting the two ends by suture, returning them into the abdomen and closing the parietal wound. The difficulties in the way of this last and perhaps most desirable method are numerous. The two ends of the gut have to be kept in accurate apposition while the sutures are being inserted. The corresponding parts of the two portions of the bowel must be precisely approximated. Any sudden movement of the parts may cause the sutures already inserted to tear out, and the relation of the divided ends to be disturbed. Moreover the intestine operated upon is lax, flabby, collapsed, and empty, and the accurate adjustment of sutures to such a structure is a matter of considerable difficulty. A still further obstacle is found in the fact that the gut above the occlusion is usually much dilated, while that below it is narrow and shrunken. The two cut ends under these circumstances are so disproportionate in size that the union of the two by suture becomes a problem somewhat difficult to solve.

From what has been said it will be seen that the following circumstances may contribute to a failure of the operation. The bowels may be unduly exposed and handled during the operation. The gut may be damaged by the clamps or ligatures applied to prevent extravasation of its contents. The difficulty of introducing the proper number of sutures may render the operation of long duration, and on this account it has on more than one occasion occupied over two hours.

Fæcal extravasation may occur after the united gut has been returned, owing to the improper manner in which the stitches have been applied, or to their insufficient number. In one instance (Baum's case of resection of part of the ascending colon) only six sutures were applied, with the result of fæcal matter escaping soon after the operation was completed. When one remembers that in cases of resection of the pylorus from forty to sixty separate stitches are not considered too many, it will be obvious that six sutures applied to the colon will be 
almost as useless as one. In several cases the fatal issue appears to have been due to a kinking or abrupt angular bending of the gut at the seat of the resection. This result can, I think, be avoided by taking care that the gut at the seat of the operation is well occupied by intestinal contents before it is returned into the abdomen. If moderately distended the gut has little opportunity for bending, while the distension serves to test the security of the stitches. In the second place, a proper treatment of the mesentery may help to prevent occlusion at the suture line. The mesentery attached to the piece to be resected should not be divided in a straight line close to its attached border as has been usually done. The better plan is to remove a triangular piece, the base of the triangle corresponding to the portion of bowel to be removed. The edges of the gap thus formed in the mesentery should then be carefully approximated by a few points of suture, and care should be taken to remove no more of the membrane than that corresponding to the segment of gut to be excised.

In two or three cases, from want of proper clamping, fæcal extravasation occurred from rupture of the gat during the necessary handling of the part, and a fatal issue was thus at once rendered almost inevitable. I have been unable to find any case of stricture of the gut following the direct suturing of the intestine after resection. In Rembard's case a piece of the suture used was found in a stool twelve days after the operation. It would appear that the sutures are soon covered over with lymph, and in a case reported by Berger this protection is said to have occurred eight hours after the operation.

That the integrity of the gut can be fully established after resection followed by suturing of the divided ends is, I imagine, proved by the cases of pyloric resection where the test is as severe as it can be. In nine experiments made upon animals by Madelung perfect union of the divided bowel occurred after the resection of a segment of the intestine. 
As the operation of resection, however, at present stands, it must be confessed that the best results have been obtained in those cases where the excision has been followed by the formation of an artificial anus; and, on the other hand, little encouragement has been offered for the performance of direct apposition of the divided ends and the replacement of the bowel within the abdomen. This latter procedure has failed from gross faults in the details of the operation, and if those faults be remedied I see no reason why this method should not surpass in its completeness and in the certainty of its results the plan that involves the formation of a temporary or permanent intestinal fistula.

In regard to resection of the colon the above remarks will, with some modifications, fully apply. The great difficulty in resection operations performed on the large intestines lies in the situation of the abdominal incision. When a distinct tumour exists and when the precise locality of the disease is obvious, it may be the best practice to cut down upon the mass in one or other loin, making use of one of the incisions for lumbar colotomy. When, however, it is impossible to diagnose the site of the disease with absolute certainty, there appears to be no alternative but to make an exploratory incision in the middle line. This has been done in two cases (Gussenbauer's and Marshall's), while in a third case (Baum's) the incision was made two inches and a half to the right of the middle line. In Baum's case the disease was in the ascending colon, in the two other cases in the descending. In each of the three instances it was found impossible to remove the mass through the median incision. In Marshall's case, therefore, a second wound was made in the loin, and in the other cases a free incision was carried transversely to the loin from the original wound. It is significant that these three cases are those that were fatal out of the seven recorded cases of colectomy, although the gut was in each instance removed finally from the lumbar region as in the non-fatal instances. 
There are many points in favour of resecting the colon from a median incision, and this applies even to cases where a mass is obvious. That it can be easily done, if proper appliances are used, is, I think, demonstrated by the case given below in which I resected without difficulty a portion of the descending colon through a wound in the middle line.

In the lumbar incision the space is limited and it has been found difficult to fully expose a large mass in that locality. In one case (Martini's) it was found to be impossible to reunite the colon after the resection, and thus a permanent artificial anus was inevitable. This difficulty would not have been encountered had the bowel been approached from the middle line. Of course if it is intended that an artificial anus shall be formed after the resection, the loin is the proper spot to select for the operation. But if it is intended to resect the bowel and then to unite the divided ends by suture, I am strongly of opinion that the median incision is the best. In the discussion that followed upon Mr. Bryant's paper read before this Society in March last, I find it reported that two surgeons, Mr. Howard Marsh and Mr. Harrison Cripps, were inclined to advise if not a median incision at least a vertical incision at the outer edge of the rectus. They contended, with I think considerable justness, that the incision in the loin did not afford sufficient room for other than very limited operations.

I will now beg to draw the attention of the Society to an appliance I have had made that will, I hope, meet many of the difficulties incident to resection.

The apparatus consists in the first place of two clamps to secure the gut above and below the site of resection. Each clamp is made of two separate and light metal bars, provided with an india-rubber pad on the surfaces that are in contact with the gut. The clamp is two inches and a half in length, and one part being placed beneath the gut and the other upon it, the two are then approximated by screws placed at each end. By this 
ON RESECTION OF PORTIONS OF INTESTINE.

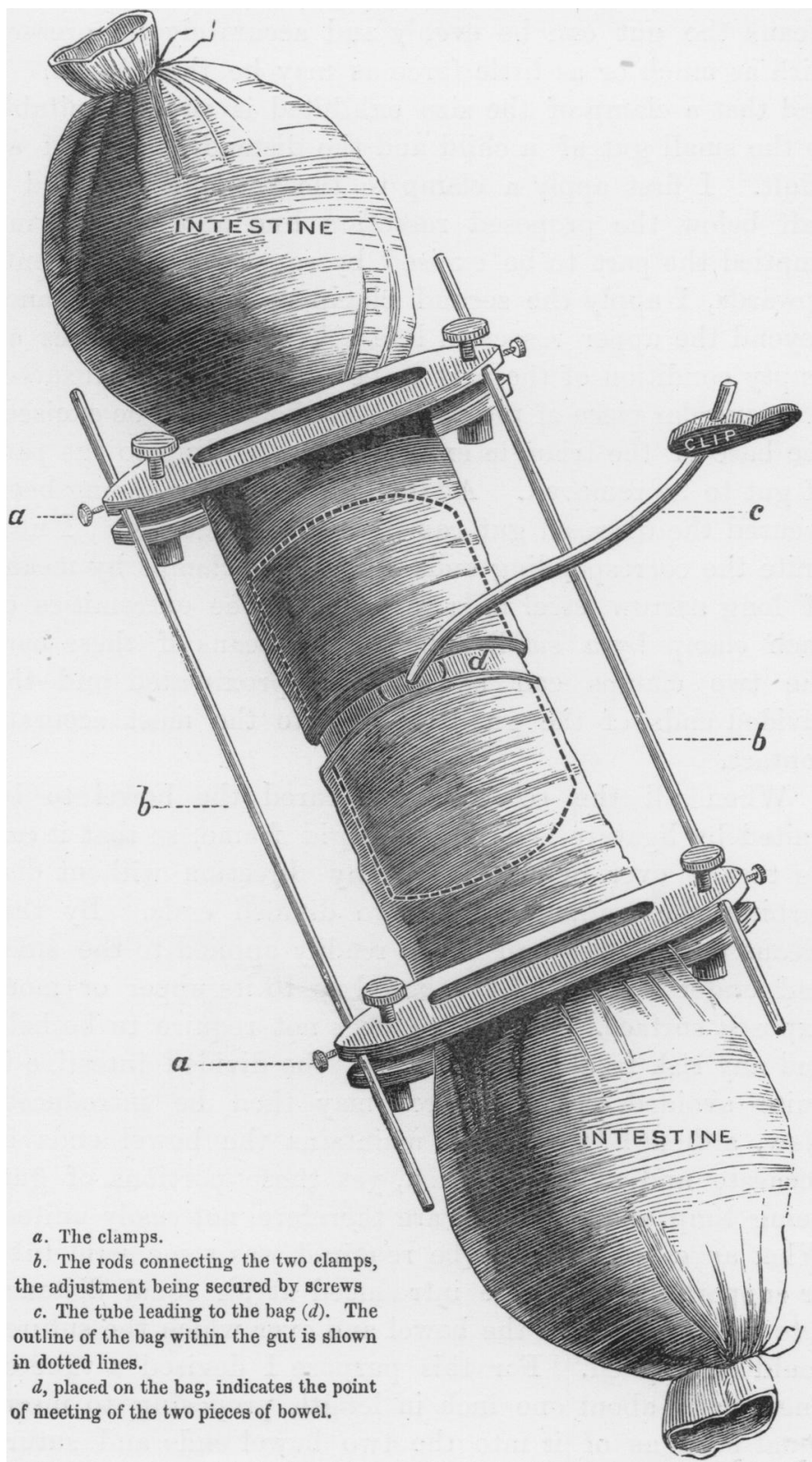


means the gut can be evenly and accurately compressed with as much or as little force as may be thought fit. I find that a clamp of the size exhibited is equally suitable to the small gut of a child and the distended colon of an adult. I first apply a clamp to the gut one inch and a half below the proposed resection line and then having emptied the part to be excised by squeezing its contents upwards, I apply the second clamp at a similar distance beyond the upper resection line. Thus in most cases an empty condition of the part to be removed can be insured. A triangular piece of the mesentery should then be excised, the base of the triangle exactly corresponding to the part of gut to be removed. All its divided vessels having been secured the diseased gut can be at once resected. I now unite the corresponding ends of the two clamps by means of long narrow steel rods secured to the extremities of each clamp by a small screw. By means of these bars the two clamps can be evenly approximated and the divided ends of the gut brought into the most accurate contact.

When all the screws are secured the bowel to be united by ligature is held in a rigid frame, so that it can be turned over or moved in any direction without disturbing the contact of the two divided ends. By this means the sutures can be as readily applied to the sides and under surface of the bowel as to its upper or more exposed surface. The frame does not require to be held and any risk from the slipping of the divided intestine is quite avoided. The sutures may then be introduced. Now, although the frame maintains the bowel ends in accurate and stable position, yet those portions of gut, being limp and collapsed, are therefore not easily united. What appeared to me to be required was some solid tube or support that could be introduced at an equal distance into the two ends of the bowel and over which the sutures could be applied. For this purpose I devised a tube of fine gelatin about one inch in length proposing to insert equal portions of it into the two bowel ends and suture 
the parts over it as over a substantial support. I imagined that the gelatin tube would after the operation prevent kinking of the gut, would lessen the risk of extravasation of the contents and would keep the tube patent. Moreover, it would possibly not be dissolved until the sutures had been rendered somewhat more secure by lymph. But to be of any use these tubes would have to be kept in a multitude of sizes, and moreover they would hardly be of any use under those common circumstances where a distended bowel above an obstructiou has to be united to a shrunken bowel below it. Discarding these tubes I had a very thin india-rubber bag made about three inches in length, of sausage shape, and that could be distended by air to a large size through a tube inserted about the middle of its long axis. Having blown out this bag till it was about the size of the divided bowel, I inserted one end into the lower piece of the intestine, and the other end into the upper piece. The tube that supplied it with air would thus occupy the suture line. After being inserted the bag can be distended to any size, and can thus quite overcome any inequalities in calibre that may exist between the two pieces of bowel. It moreover forms a firm support in the interior of the gut over which the sutures can be most readily applied. If the distension of the bag disturbs the mutual relations of the divided ends, this can be rectified by a slight readjustment of the clamp. The sutures are then applied all round the gut and almost close up to the interruption in the suture line caused by the tube that fills the bag. The sutures having been applied, the bag is exhausted of air and is withdrawn from the bowel, and so thin is its structure that the collapsed bag can be drawn through a hole with the circumference of a No. 13 catheter. It only remains, then, to close the little opening left after the removal of the bag. If the stitches are properly applied, there should not be the least risk of pricking the bag, for the sutures should not include the mucous membrane. Of the various forms of suture, viz. Gely's, Czerny's, Güssenbauer's, and Lem- 
bert's, I think that the last named is to be preferred as it is the most easy to introduce, and in this operation I imagine that time is a matter of great moment. At least from thirteen to twenty points of suture should be employed. Madelung advises that they should not be more than three millimètres apart. The best material for the stitch is, I believe, the finest "Chinese twist," and in using Lembert's suture I have found a small curved needle used with a needle holder the most convenient. Before the gut is replaced in the abdomen and the wound in the parietes closed, it is necessary that the two cut edges of the mesentery be united by a few points of suture.

I will conclude this paper by very briefly detailing a case of resection of a part of the descending colon. This is the third time, I believe, that the operation has been performed in this country, and it is, so far as I am aware, the only instance on record where a portion of the colon was excised through an incision in the middle line. The patient I might state was in a very prostrate condition at the time of the operation and died in twelve hours.

The patient, a sailor, æt. 56, was admitted into the London Hospital under the care of my colleague, Dr. Warner, on September 1st. 1882. There was nothing of note in the details of his previous history. For two months before admission he had suffered from occasional attacks of colic associated with slight vomiting and absolute constipation, and then followed by diarrhœe. On admission he was feeble and was losing flesh, his face was contracted and his eyes sunken. He could take but little food and vomited a great deal of what he did take. His bowels were as a rule constipated, but were relieved occasionally by a species of diarrhœa. He complained of repeated attacks of severe abdominal pain associated with movement of the bowel that could be felt by the hand. The abdomen was moderately and evenly distended; there was no abdominal tumour and nothing to be felt per rectum. On an enema being given $4 \frac{1}{2}$ pints were easily introduced before any returned. These symptoms continued with 
some modifications until September 12th; the man in the mean time becoming feebler and more wasted. On this date he became much worse, the vomiting was severe and continuous, the pain constant, and the abdomen more distended. When I saw him on September 14th, he was extremely feeble and much exhausted, and troubled by an almost constant vomiting that, however, was never stercoraceous. I had ventured to diagnose a stricture of the bowel but could find noindications as to its probable site. At 10 p.m. the man was anæsthetised and the abdomen once more very carefully examined with the same negative results. I then made an incision in the middle line below the umbilicus. The first part of the intestine to present was a greatly distended cæcum. Introducing the hand into the abdomen I proceeded to examine the whole length of the colon and soon found a small hard mass in the descending colon about two inches or so below the splenic flexure. To fully expose it, the small intestines were allowed to protrude. They were deeply congested and presented a vast number of blood-extravasations under their peritoneal covering. They were carefully protected by warm carbolised cloths but I feel sure that to allow the escape of these parts was a mistake in the procedure that may, and should have been avoided.

With a blunt director I divided the peritoneum that was reflected from the descending colon to the parietes and with some little manipulation dragged the strictured bowel towards the middle line. I soon had freed it sufficiently to allow of one clamp being applied above the seat of disease, the other clamp having been already applied to the gut below. I divided the peritoneal connection of the part to be removed, resected it and applied the sutures to the divided ends of the bowel in the manner already described. The frame answered admirably and without its use I believe that the resection would have been impossible owing to the depth at which one had to work. 'T'he clamps, frame, and distending bag were all employed in this case. I introduced twenty-five sutures after Lembert's method, bringing vOL. LXVI. 
the two serous surfaces together and avoiding the mucous membrane with the needle. The clamps were then removed, and the fæcal matter in the distended gut above the resection line was squeezed down so as to occupy the part just operated upon. I was glad to find that.even when pressure was applied to the part above no escape from the gut of its contents occurred at the line of the sutures. The bowel was replaced and the abdominal wound closed. The operation took one hour and a quarter and was performed under strict antiseptic precautions.

At the autopsy there was commencing general peritonitis. There was no trace of blood in the peritoneal cavity and not the least escape of fæcal matter at the site of the operation. The colon at the line of the sutures was distended with fluid fæces so that it was as large as the engorged bowel above it. A quantity of fæcal matter had likewise found its way into the rectum. The stricture was composed of a dense mass about the size of a walnut. It surrounded the bowel equally at all parts, and had so narrowed its lumen that the tube would barely admit the tip of the little finger. It proved on examination to be a cylindrical epithelioma.

It is much to be regretted that the operation was not performed sooner, for the patient was in so feeble a condition when anæsthetised that his recovery from even a milder operation would have been a matter of question. It would possibly have been better if in this patient I had adopted the excellent practice carried out by Mr. Pitts in a like case. $^{1}$ Mr. Pitts-in a case of stricture of the descending colon, that could not be diagnosed before the abdomen was opened-made a median incision, discovered the mass and then performed colotomy in the left loin, proposing to excise the mass at a future period. His patient made a good recovery. It is extremely doubtful, however, if my. patient could have borne this double operation.

The case-so far as it goes-appears to demonstrate

1 'St. Thomas's Hospital Rerorts,' vol. ii, 1882, p. 75. 
ON RESECTION OF PORTIONS OF INTESTINE. 67

that with proper appliances a portion of the colon can be resected through a median incision, and that the gut can be so united after the excision of a portion as to quite prevent any escape of the intestinal contents. 